\title{
Transcriptome-based phylogeny and whole-genome duplication in Theaceae
}

Qiong Zhang ${ }^{\text {a, b }}$, Lei Zhao ${ }^{\text {, }}$ Jian-Li Zhao ${ }^{\text {d }}$, Ryan A. Folk ${ }^{\mathrm{e}}$, Nelson Zamora ${ }^{\mathrm{f}}$, Pamela S. Soltis ${ }^{\mathrm{g}}$, Douglas E. Soltis ${ }^{\mathrm{g}}$, Shi-Xiong Yang ${ }^{\mathrm{a}}$, Lian-Ming Gao ${ }^{\mathrm{a}}$, Hua Peng ${ }^{\mathrm{a}^{*}}$, Xiang-Qin $\mathrm{Yu}^{\mathrm{a}}{ }^{*}$

${ }^{a}$ CAS Key Laboratory for Plant Diversity and Biogeography of East Asia, Kunming Institute of Botany, Chinese Academy of Sciences, Kunming 650201, China;

${ }^{\mathrm{b}}$ College of Life Sciences, University of Chinese Academy of Sciences, Beijing 100049, China;

${ }^{c}$ Germplasm Bank of Wild Species, Kunming Institute of Botany, Chinese Academy of Sciences, Kunming, Yunnan 650201, China;

d Yunnan Key Laboratory of Plant Reproductive Adaption and Evolutionary Ecology, Yunnan University, Kunming 650091, China;

e Department of Biological Sciences, Mississippi State University, MS 39762, United States;

${ }^{\mathrm{f}}$ National Herbarium of Costa Rica (CR), Natural History Department of National Museum of Costa Rica, San José, Costa Rica;

g Florida Museum of Natural History, University of Florida, Gainesville, FL 32611, United States

* Correspondence:

Xiang-Qin Yu, yuxiangqin@mail.kib.ac.cn

Hua Peng, hpeng@mail.kib.ac.cn 


\begin{abstract}
Theaceae, with three tribes and nine genera, is a family of great economic and ecological importance. Recent phylogenetic analyses based on plastid genome resolved the relationship among three tribes and the intergeneric relationships within Gordonieae and Stewartieae. However, generic level relationships within the largest tribe Theeae were not fully resolved and potential hybridization among genera within Theeae revealed previously also remains to be tested further. Here we conducted a comprehensive phylogenomic study of Theaceae based on transcriptomes and low-depth whole-genome sequencing of 57 species as well as additional plastome sequence data from previous work. Phylogenetic analyses suggested that Stewartieae was the first-diverging clade in Theaceae, consistent with previous study using plastomic data. Within Theeae, the highly supported Apterosperma-Laplacea clade grouped with Pyrenaria with maximum support based on the partitioned and unpartitioned concatenation analyses using the 610 low-copy nuclear genes, leaving Camellia and Polyspora as another sister genera in the tribe. PhyloNet analyses suggested one reticulation event within Camellia and Pyrenaria respectively, but no intergeneric reticulations were detected in Theeae. Another introgression was found between Gordonia lasianthus and the common ancestor of Gordonieae during the Late Oligocene. The existing land bridges (e.g. Bering land bridge) might have facilitated this ancient introgression. Further researches need to be conducted to uncover the interspecific introgression pattern within Camellia. Ks distribution analyses supported the tea family shared one whole-genome duplication (WGD) event Ad- $\beta$, which was recently mapped to the clade containing core Ericales, Primuloids, Polemonioids and Lecythidaceae.
\end{abstract}

Keywords: Theaceae, phylogeny, transcriptome, low-copy nuclear genes, phylogenetic network 


\section{Introduction}

Theaceae consists of nine genera in three tribes and contains more than 200 species of evergreen and deciduous trees and shrubs that are disjunctly distributed in temperate, subtropical and tropical areas of eastern to southeastern Asia, and eastern North America to Central and South America, i.e. an Amphi-Pacific disjunction (Kobuski, 1949; Kobuski, 1950; Prince, 1993; Stevens, 2001 onwards; Min \& Bartholomew, 2007). Members of Theaceae have great economic and ecological importance, including familiar plants such as tea (e.g. C. sinensis (L.) Kuntze), oil plants (e.g. C. oleifera C. Abel) and a number of woody ornamentals (e.g. Camellia japonica L., C. reticulata Lindley), and tree representatives (e.g. Schima) and smaller woody lineages (e.g. Camellia, Stewartia) are dominant or common species of the subtropical evergreen broadleaved forests in East Asia (Tang, 2015). Due to the excessive collections and habitat destruction, several species mainly from Camellia have been listed as (critically) endangered, such as $C$. fangchengensis S. Ye Liang \& Y. C. Zhong, C. hekouensis C. J. Wang \& G. S. Fan, C. piquetiana (Pierre) Sealy (IUCN, 2020). Meanwhile, many new species (Orel, 2006; Orel \& Wilson, 2010b; Orel \& Wilson, 2012; Orel et al., 2013; Lee \& Yang, 2019; Liu et al., 2019), and subgeneric taxa (Orel \& Wilson, 2010a; Orel et al., 2014) have been described and published for Camellia in the past ten years.

After the establishment of Theaceae in 1813 (Mirbel, 1813), the systematic boundaries of genera in the family as defined by morphology has changed significantly (Bentham \& Hooker, 1862; Melchior, 1925; Takhtajan, 1997), from two genera to six tribes and 32 genera. Molecular systematic studies recognized three tribes and nine genera (APG I, 1998; APG II, 2003; APG III, 2009; APG IV, 2016). Since then, a number of systematic studies using DNA markers, 
morphology, anatomy and cytology have been conducted to explore the relationships among tribes and genera (Ye, 1990; Tsou, 1998; Prince \& Parks, 2001; Yang et al., 2004; Wang et al., 2006; Yang et al., 2006; Li et al., 2013; Zhang et al., 2014; Yu et al., 2017b). However, many unresolved phylogenetic relationships remain in this family.

Firstly, phylogenetic relationships among the three tribes in Theaceae remain controversial. Evidence from floral development indicated a close relationship between the tribes Gordonieae and Stewartieae (Tsou, 1998; treated as subtribes in this study), which was supported by phylogenetics based on small single-copy region (SSC) of the plastome with conflicting support between non-model-based maximum-parsimony (MP), model-based maximum-likelihood (ML) and Bayesian inference (BI) analyses (Li et al., 2013). However, an analysis of 46 morphological characters supported sister between Gordonieae (=Schimeae) and Theeae (Wang et al., 2006), which was supported by phylogenetics of plastid $r b c L$, mat $K$ and $t r n L-F$, mitochondrial matR and nrITS (Prince \& Parks, 2001; Yang et al., 2004; Yang et al., 2006) and the whole plastome sequences (Yu et al., 2017b). In a recently published phylogenetic context of Ericales based on 25 genomic loci from the plastid, nuclear and mitochondrial genomes, Theeae and Gordonieae were also recovered as sister with Stewartieae as the first-diverging clade (Rose et al., 2018). Given conflict and uncertain support, additional nuclear genes are needed to draw a more comprehensive conclusion.

Moreover, intergeneric relationships within Theeae have been challenging to resolve, especially for the phylogenetic position of Apterosperma and Laplacea. Since the time of its original description (Chang, 1976), Apterosperma has been formally placed in tribe Gordonieae (=Schimeae) and considered close to Schima and Franklinia based on similar morphological characters (Ye, 1990; Chang \& Ren, 1998; Tsou, 1998). A combined molecular phylogenetic 
analysis based on nrITS, plastid trnL-F, and mtDNA matR sequence data placed Apterosperma as the first-diverging lineage within Theeae (Yang et al., 2004). Based on analyses of 46 morphological characters, Apterosperma was also placed as the first-diverging lineage within Theeae (Wang et al., 2006). Using five genomic regions (chloroplast: atpI-H, matK, psbA5'R$A L S-11 F$, rbcL; nuclear: $L E A F Y$ ) and 30 species representing four of the five genera within Theeae, Zhang et al. (2014) found that Apterosperma formed a sister relationship with Polyspora in the cpDNA tree, but was placed within a clade comprising Tutcheria (=Pyrenaria) and Parapyrenaria (=Pyrenaria) in the LEAFY tree. In their study, Camellia and Pyrenaria were not monophyletic, and inconsistent phylogenetic placement of some species between the nuclear and chloroplast trees were proposed to be the result of widespread hybridization among genera in Theeae. In contrast to Apterosperma, very few studies have included Laplacea. The study of Prince \& Parks (2001) recovered Laplacea within a clade comprising Camellia, Tutcheria (=Pyrenaria) and Glyptocarpa (=Pyrenaria). In our previous study, Apterosperma and Laplacea were sister with strong support (MLBS=96\%, BIPP=1.0). However, Apterosperma-Laplacea clade grouped either with Camellia-Polyspora or with Pyrenaria with moderate support based on different partitions of the plastome, grouped with Polyspora with weak support from nrITS dataset, or even as the early branching clade from the combined plastome and nrITS dateset (Yu et al., 2017b).

Whole genome duplication (WGD), an important evolutionary force, was reported to occur in the common ancestor of extant seed plants, extant angiosperms and core eudicots (Ad- $\gamma$ ) (Jiao et al., 2011; Vekemans et al., 2012). WGD events were also found in the early history of numerous families such as Asteraceae, Brassicaceae, Fabaceae, Poaceae and Rosaceae (Huang et al., 2016; Xiang et al., 2017; Qiao et al., 2019). Based on the kiwifruit (Actinidia chinensis) genome, 
researchers proposed a WGD event called Ad- $\beta$, which was shared by Actinidia and Camellia (Shi et al., 2010; Huang et al., 2013). However, using genome collinearity and also MAPS pipeline, Ad- $\beta$ was recently mapped to the core Ericales (Leebens-Mack et al., 2019), and more specifically to the clade comprising core Ericales, Primuloids, Polemonioids and Lecythidaceae (Zhang et al., 2020). In Theaceae, whole genome was only available for Camellia, and results for WGD event from previous studies were incompatible (Xia et al., 2017; Wei et al., 2018; Xia et al., 2020), and need more evidence and broaden sampling.

RNA-seq (transcriptomics) is becoming an important approach for plant phylogenomics, (Soltis et al., 2013; Yang \& Smith, 2013; Wickett et al., 2014; Huang et al., 2016; Yu et al., 2018; Zhang et al., 2020), and has been widely used to explore the origin and early diversification of land plants (Wickett et al., 2014), deep-level (among eight clades) angiosperm phylogeny (Zeng et al., 2014), phylogenetic relationship within eudicot, asterids and rosids (Zhao et al., 2016; Zeng et al., 2017; Zhang et al., 2020), and intergeneric relationships for several species-rich orders or families (Caryophyllales, Asteraceae, Brassicaceae, Rosaceae and Pinaceae) (Huang et al., 2015; Yang et al., 2015; Huang et al., 2016; Xiang et al., 2017; Ran et al., 2018). Additionally, the 1000 Plant Genomes Project (1KP), sequencing transcriptomes from 1124 species representing the diversity of green plants, provided resolution of much phylogenetic uncertainties across the green tree of life (Matasci et al., 2014; Leebens-Mack et al., 2019). Furthermore, combining evidence from plastid and nuclear genomic compartments allows the detection of cytoplasmic introgression and other forms of hybridization (Calvo et al., 2013; Folk et al., 2017; Guo et al., 2018; Morales-Briones et al., 2018; Stubbs et al., 2020).

Here, transcriptomes/low-depth whole-genome sequencing of 57 species within Theaceae were sequenced, and both nuclear and plastid genes were extracted. Integrated with plastome 
data from our previous study, firstly we aim to reconstruct the nuclear phylogenetic framework of the tea family, to illuminate the relationships among tribes and genera and the phylogenetic position of Apterosperma and Laplacea. Furthermore, we also test the intergeneric hybridization hypothesis proposed by previous work, and investigate whether previous WGD found in Camellia was shared by other genera of Theaceae.

\section{Materials and Methods}

\section{Taxon sampling, RNA sequencing and whole-genome sequencing}

We collected 58 samples representing 57 species, three tribes and all nine genera of Theaceae

(Table 1). Fresh, mature and healthy leaves were collected and then frozen immediately in liquid nitrogen. For some of the samples, we stored the fresh leaves in a $-80^{\circ} \mathrm{C}$ freezer after field collection. Total RNA was extracted from the frozen leaves using the Spectrum ${ }^{\mathrm{TM}}$ Plant Total RNA Extraction Kit (Sigma). Silica-dried leaves of two species (Stewartia malacodendron and Laplacea fruticosa) were used for low-depth whole-genome sequencing (ca. 30×). RNA-Seq and low-depth whole-genome sequencing library construction, Illumina HiseqXten sequencing, raw data cleaning and quality control were performed at Novogene, China. Additionally, chloroplast genome data were obtained from our previous studies (Yu et al., 2017a; Yu et al., 2017b). Six species from Symplocaceae, Styracaceae, Pentaphylacaceae and Diapensiaceae were also used as outgroup taxa. The transcriptome data of these six species were downloaded from GenBank. Based on the results from previous phylogenetic studies of Theacaee, Pyrenaria and Stewartia were treated in the broad sense according to the treatment in the Flora of China (Min \& Bartholomew, 2007). In our previous study (Yu et al., 2017b), we found that Laplacea grandis 
grouped with Gordonia lasianthus and should therefore be removed into Gordonia, thus here we use the name Gordonia brandegeei $\mathrm{H}$. Keng (=Laplacea grandis) according to Keng (1980).

\section{Sequence assembly and ortholog identification}

Quality control of the raw sequencing reads was performed using the Fastp version 0.20.1, adapter, reads containing $\mathrm{N}$ and reads with low quality score (percentage of base $<=\mathrm{Q} 20$ ) were removed. Trinity v2.8.4 was used to conduct de novo assembly of cleaned Illumina RNA-Seq reads of each species (Grabherr et al., 2011; Haas et al., 2013). TransDecoder v5.3.0 was used to identify candidate coding regions with default parameters. ORFs (open reading frames) with a minimum length of 100 amino acids (AA) were used for further analyses. CD-HIT v4.6 (Li \& Godzik, 2006) was used to remove redundant contigs with a threshold of 0.99. Orthogroups (OG) were identified and filtered following the pipelines proposed by Yang \& Smith (2014). For the data obtained from low-depth whole-genome sequencing, we first de novo assembled the genome using Platanus (http://platanus.bio.titech.ac.jp/platanus-assembler/platanus-1-2-4) with default parameters. Then RepeatMasker and RepeatModeler (http://www.repeatmasker.org/) were performed to identify tandem repeats and TEs. We carried out gene annotation using de novo gene prediction (AUGUSTUS, http://evidencemodeler.github.io/) and homolog prediction (Exonerate, https://www.ebi.ac.uk/about/vertebrate-genomics/software/exonerate), then generated an integrated gene set using EVidenceModeler (http://evidencemodeler.github.io/). Preliminary gene trees were reconstructed using RAxML v8.2.12 (Stamatakis, 2014). To reduce potentially misidentified orthologs, we examined single-gene trees of the 631 OGs and found three tribes within Theaceae was not monophyletic in 21 gene trees. Because all three tribes within Theaceae was consistently monophyletic in previous studies (Prince \& Parks, 2001; Yang 
et al., 2004; Li et al., 2013; Yu et al., 2017b), these 21 OGs likely contain hidden paralogs and are therefore problematic for downstream analysis. Therefore, we excluded the 21 OGs to yield a final gene data set with 610 OGs (hereafter refer to the reduced 610 OGs dataset). For those species without chloroplast genome data, we first assembled the chloroplast genome using GetOrganelle v1.6.2e (Jin et al., 2020) and the protein-coding sequences (CDS) were extracted from the transcriptome and whole-genome sequencing data. For those species with already complete chloroplast genomes, protein-coding sequences were extracted following parallel methods to yield a combined matrix.

\section{Phylogenetic analyses and evolutionary network}

The obtained nucleotide sequences were aligned with MAFFT v7.407 (Katoh \& Standley, 2013). Alignment statistics were calculated by AMAS (Borowiec, 2016). Both concatenation and coalescent methods were used to reconstruct intergeneric relationships of Theaceae. For concatenation, partitioned and unpartitioned Maximum-Likelihood analyses and Bayesian inference (BI) were performed by using RAxML v8.2.12 (Stamatakis, 2014) and MrBayes v3.2.6 (Ronquist et al., 2012). In the unpartitioned ML analysis, GTRGAMMA model was used and bootstrap support (BS) value were calculated using 1000 replicates. In the unpartitioned BI analysis, parameters were set to $n s t=6$ and rates=gamma. Four chains were run for $2,000,000$ generations with random initial trees, every 100 generations were sampled and the first $25 \%$ of the samples were discarded as burn-in. In the partitioned analyses, partitioning schemes and models were selected by using PartitionFinder version 2.1.1 for 610 OGs (Lanfear et al., 2016) and 188 subsets were obtained for the CDS dataset. For coalescent analysis, a ML gene tree was reconstructed for each LCO with RAxML using the same parameter settings as above. The best 
ML gene trees and 100 bootstrap replicate trees generated from each LCO were used to estimate species tree and supporting values in ASTRAL v5.6.3 (Mirarab et al., 2014). Support of the ASTRAL-II species tree was quantified using the local posterior probability (LPP) of a branch as a function of its normalized quartet support (Sayyari \& Mirarab, 2016). We used PhyParts (Smith et al., 2015) to examine patterns of gene tree concordance and conflict within the nuclear genome and to reveal subsets of the nuclear genome supporting alternative relationships in the plastid topology, visualizing the results using the program phypartspiecharts (https://github.com/mossmatters/phyloscripts/). To test the previous hypothesis of intergeneric hybridization, we used PhyloNet v3.8.0 (Than et al., 2008) to infer an evolutionary network for Theaceae, using the command "InferNetwork_MPL" under a maximum pseudo-likelihood framework (Yu \& Nakhleh, 2015) and 505 individual gene trees. Given that the computational time needed by network methods scales very rapidly with taxon number (Folk et al., 2018), we reduced the sampling to a computationally tractable size (22 species in total, fewer than 30 taxa), taking one (i.e. Apterosperma, Franklinia and Laplacea) to six (i.e. Camellia) species of each genus, and only one outgroup species (different outgroup species were selected for each gene tree because of missing data) (Table 2). The maximum pseudo-likelihood algorithm requires $a$ priori specification of the number of reticulating branches; the number of reticulations were set as one, two, three and four in repeated analyses per developer recommendations. Gene trees with 
branches $<50 \%$ BS were collapsed, and five optimal networks returned for each analysis. The command CalGTProb was used to compute the likelihood scores and select the best network.

\section{Whole genome duplication analysis}

In order to investigate the ancient whole-genome duplications (WGD) in Theaceae, we applied the Python package "wgd" (Zwaenepoel \& Van de Peer, 2019) to construct the synonymous substitutions $(K s)$ distributions (ranging from 0.05 to 3 ) among paralogs from 56 Theaceae transcriptomes and six outgroup transcriptomes. Using the command "mcl" to blast and cluster sequences with each CDS, the command "ksd" and "mix" was used to construct the Ks distribution and mixture modeling of $K$ s distributions, respectively. For the analysis of the mixture model, we chose the method BGMM in wgd package.

\section{Results}

\section{Characteristics of transcriptomes and datasets}

We sequenced 5.85 to $10.6 \mathrm{~Gb}$ of transcriptome data from 56 individuals of 55 species, and 138.7 and 165.7 Gb whole-genome sequencing data for each of Laplacea fruticosa and Stewartia malacodendron. In total, our phylogenetic analysis represented 58 individuals of 57 species from all nine genera and three tribes of Theaceae (Table 1). To construct the plastid matrix, plastid protein-coding genes from 27 species were extracted from the transcriptome or whole-genome sequencing data generated here, while plastid genome from 31 species were obtained from complete chloroplast genomes already available from our previous study. In total, 610 OGs were obtained from 56 transcriptomes and two whole-genome sequencing datasets, with the aligned length ranging from $309 \mathrm{bp}$ to $8,854 \mathrm{bp}$ and missing data percentage from $0 \%$ to $29.65 \%$. The 
aligned length of the concatenated 610 OGs was 858,606 bp, with 227,708 (26.5\%) variable sites, $117,025(13.6 \%)$ parsimony informative sites and $21.67 \%$ missing data. The alignment length of the concatenated 80 plastid coding genes was $69,225 \mathrm{bp}$, with 7,236 (10.5\%) variable sites, 3,695 (5.3\%) parsimony informative sites and $11.88 \%$ missing data.

\section{Phylogenetic relationships and network within Theaceae}

The topology recovered from both partitioned and unpartitioned RAxML analyses based on the concatenated 610 low-copy nuclear genes strongly supported the sister relationship between Theeae and Gordonieae (MLBS=100\%, PP=1.00, Fig 1, Fig S1). Additionally, based on Phyparts analysis of 509 low-copy nuclear genes, 408 gene trees $(80.2 \%)$ supported the above topology (Fig. 2). Coalescent-based species tree inferred from ASTRAL yielded a concordant relationship among the three tribes with the concatenation analysis (LPP=1.00, Fig S3). RAxML analyses based on the 80 protein-coding genes of the plastid genome also supported a sister relationship between Theeae and Gordonieae $(\mathrm{MLBS}=100 \%, \mathrm{PP}=1.00$, Fig S3). Hence support was strong and unanimous across data partitions and analytical methods employed here.

Generic level relationships recovered within Gordonieae and Stewartieae were largely consistent with previous phylogenetic studies of Theaceae. Within Theeae, the sister relationships between Camellia and Polyspora and between Apterosperma and Laplacea fruticosa were both maximumly supported in all of the analyses using trascriptomic data (Fig. 1, Fig. 2, Fig. S2, Fig. S3). Nevertheless, the position of the Apterosperma-Laplacea clade changed between different analyses. In both of the partitioned and unpartitioned concatenation analyses using the 610 low-copy nuclear genes, the Apterosperma-Laplacea clade grouped with Pyrenaria with moderate support $(\mathrm{MLBS}=72 \%, \mathrm{PP}=1.00$; Fig. 1 ; MLBS=67\%, PP=1.00; Fig. 
S2). The ASTRAL topology (Fig. S3), while largely congruent overall with the results from concatenated analyses (Fig. 1, Fig. S2), indicated that Apterosperma-Laplacea clade was weakly supported as sister to Camellia-Polyspora clade (LPP=0.38, Fig. S3). At lower taxonomic levels, PhyParts recovered strong discordance across many parts of the tree, with only 95 gene trees supporting the sister relationship between the Apterosperma-Laplacea clade and the CamelliaPolyspora clade, and 408 gene trees support conflicting/alternative resolutions (Fig. 2). Phylogenetic trees based on the plastid 80 protein-coding genes dataset were highly consistent with our previous study with the exception that Apterosperma was sister to Camellia (MLBS=86\%, PP=1.00, Fig. S1), and Laplacea fruticosa grouped with Pyrenaria with weak support (MLBS=62\%, PP=0.93, Fig. S1).

For the PhyloNet analyses, the inferred network with the highest log pseudo-likelihood (379.6899) included three reticulations (Table 2; Fig. 3). This analysis suggested one reticulation for the clade comprising Pyrenaria spectabilis var. spectabilis and Pyrenaria jonquieriana subsp. multisepala, with contribution from Pyrenaria oblongicarpa and the ancestor of Pyrenaria. Camellia tsingpienensis was recovered as a hybrid between Camellia fluviatilis and Camellia huana. It also recovered a reticulation event suggesting that Gordonia brandegeei (previously difficult to place and treated as a species of Laplacea) descends from Gordonia lasianthus and the common ancestor of Gordonia, Franklinia, and Schima (i.e. Gordonieae). All 
other analyses (reticulation numbers $=1,2,3$ ), for which likelihood was suboptimal, only detected intergeneric reticulation within Camellia (Fig. 3).

\section{Whole genome duplication}

$K_{\mathrm{S}}$ (synonymous substitution rate) analyses using all 56 ingroup transcriptomes suggested that all except one species from Theaceae presented a peak at around 0.4 (Fig. 4, Fig. S4), which is consistent with one of the WGD $(K s=0.36)$ reported in tea tree (Camellia sinensis var. sinensis) genome. No Ks peak was found in Stewartia ovata, this might be due to the stochastic error, or because the transcriptome data only represent the expressed mRNA in the tissues (e.g. roots, leaves, flowers) sampled. For six outgroup species, consistent $K s$ peaks were found at around 0.4 (Figure S4), indicating Symplocaceae, Styracaceae, Pentaphylacaceae and Diapensiaceae shared this WGD event (i.e. Ad- $\beta$ ) with Theaceae.

\section{Discussion}

\section{Relationships among tribes}

Previous phylogenetic studies have not been able to conclusively elucidate the relationships among the three tribes within Theaceae, due in part to incomplete taxon sampling and few loci, whether based on a few plastid and nuclear loci or half of the SSC of the plastid genome (Prince \& Parks, 2001; Yang et al., 2004; Yang et al., 2006; Li et al., 2013). Using 25 genomic loci from plastid, nuclear and mitochondrial genome from 4,531 species from Ericales, Theeae and Gordonieae grouped together (MLBS>70\%, PP>0.95) with Stewartieae as the first-diverging clade in Theaceae, but only 10 species were included in their study (Rose et al., 2018). Our previous study supported a sister relationship between Theeae and Gordonieae (MLBS=91\%, 
$\mathrm{PP}=1.00)$ based on the combined plastome and nuclear ribosomal DNA dataset (Yu et al., 2017b). Here we present the phylogenetic framework of Theaceae using 610 orthologous lowcopy nuclear genes. The obtained topology from both of the concatenation and coalescence analyses of the 610 low-copy nuclear genes consistently supported a sister relationship between Theeae and Gordonieae (MLBS=100\%, PP=1.00, LPP=1.00, Fig. 1, Fig. S2), consistent with the results from plastid genome $(\mathrm{MLBS}=91 \%, \mathrm{PP}=1.00$, Fig. S3). In addition, 408 among 509 lowcopy nuclear gene trees from Phyparts analysis supported the above topology (Fig. 2). The ((Gordonieae, Theeae) Stewartieae) relationship was consistent with the evolutionary pattern of the endosperm in Theaceae, as discussed in our previous study (Yu et al., 2017b).

\section{Intergeneric relationships}

The intergeneric relationships within Gordonieae and Stewartieae have been fully resolved in previous studies (Prince \& Parks, 2001; Yang et al., 2004; Yang et al., 2006; Li et al., 2013; Yu et al., 2017b). However, the relationships among the five genera in Theeae have been controversial. Laplacea falled into the clade comprising Camellia, Tutcheria (=Pyrenaria) and Glyptocarpa (=Pyrenaria) (Prince \& Parks, 2001). Zhang et al. (2014) revealed that Apterosperma formed a sister relationship with Polyspora (MLBS $=73 \%, \mathrm{PP}=1.00$ ) in the cpDNA tree, but these two genera were placed in a clade comprising Tutcheria (=Pyrenaria) and Parapyrenaria $(=$ Pyrenaria $)(\mathrm{MLBS}=68 \%, \mathrm{PP}=0.72)$ in the $L E A F Y$ tree. Based on the 610 lowcopy nuclear genes, the resolution of the relationships among the five genera in Theeae has been substantially improved. the Apterosperma-Laplacea clade received maximum support in both of the partitioned and unpartitioned concatenation analyses using the 610 low-copy nuclear genes, and grouped with Pyrenaria with $100 \%$ support (Fig. 1, Fig. S1). Although the ASTRAL 
topology suggested Apterosperma-Laplacea clade was weakly supported as sister to CamelliaPolyspora clade (LPP=0.38, Fig. S2), only 95 out of 509 nuclear genes supported this topology

(Fig. 2). The strongly supported Apterosperma-Laplacea clade also grouped with Pyrenaria with moderate support based on the whole plastid genome dataset (MLBS=67\%), the SSC (small single-copy region, $\mathrm{MLBS}=80 \%$ ) dataset and the protein-coding gene dataset (MLBS=75\%) from our previous study (Yu et al., 2017b). Taken the evidence from plastid genome and trascriptome data, we suggest (((Apterosperma-Laplacea), Pyrenaria), (Camellia-Polyspora $))$ as the most likely topology.

\section{Phylogenetic network inference}

In the study of Zhang et al. (2014), Camellia and Pyrenaria were not monophyletic, and widespread hybridization among genera in Theeae was proposed. Phylogenetic conflicts found in Stewartia were also suggested to be caused by ancient introgressive hybridization following species diversification, leading to diverging histories in the nuclear and plastid genomes (Lin $e t$ al., 2019). However, while our PhyloNet analyses supported the presence of hybridization in the history of Theaceae, it did not support the specific reticulation scenario suggested by Zhang et al. (2014); Camellia and Pyrenaria were supported as monophyletic based on the 610 low-copy nuclear genes (Fig. 1, Fig. S1). The best fit network, with three reticulation events in total, suggested two reticulation events within Camellia and Pyrenaria respectively (but no intergeneric reticulations in Theeae), and another between Gordonia lasianthus and the common ancestor of Gordonia, Franklinia, and Schima (i.e. Gordonieae). One possibility for disagreement between our work and studies could be species misidentification in the study of Zhang et al. (2014), but further work is needed to address hybridization more comprehensively. 
All other PhyloNet analyses (reticulations=1, 2, 3) had aspects similar to the full four-reticulation analyses, suggests a clear pattern of intrageneric gene flow within Camellia.

Under the favored scenario involving Gordonieae, this would suggest a minimum date of reticulation during Late Oligocene, ca. 26.2 million years ago (Ma; 95\%HPD=23.3-32.2 Ma) given a recent molecular dating analysis of Theaceae (Yu et al., 2017b). This is a plausible scenario as land bridges (e.g. Bering land bridge) existed during Late Oligocene, the eastern Asia and eastern North American flora was likely continuous across high latitude of Northern hemisphere (Tiffney, 1985; Tiffney \& Manchester, 2001; Milne, 2006), allowing for species contact and opportunities for hybridization. Fossils of Theaceae are known from high latitude Northern hemisphere localities such as North America and Germany during Ecocene and Oligocene (Grote \& Dilcher, 1992; Kvacek \& Walther, 1998; Wilde \& Frankenhauser, 1998; Kvaček, 2004). Overall, our work, while differing from previous studies in the specific scenario, supports ancient introgression at tribe level within Theaceae that is consistent with biogeographic patterns of the group.

Previous studies have likewise found evidence of hybridization event in the genus. Firstly, under artificial conditions, cultivated ornamental camellias descending from hybridization have been widely used in horticulture (Nishimoto et al., 2003; Tanaka et al., 2005; Xu et al., 2018). Secondly, under natural conditions, Cambod tea (cultivated tea of $C$. sinensis var. assamica) was suggested to have originated through hybridization between different tea types

(Meegahakumbura et al., 2016). Gene introgression was also detected between the cultivated $C$. sinensis var. assamica and C. taliensis, and C.taliensis has been suggested to be genetically involved in the domestication of C.sinensis var. assamica (Li et al., 2015). All of our PhyloNet analyses consistently supported reticulation events within Camellia (Fig. 3). For the best-fit 
scenario, Camellia tsingpienensis (Guangxi and SE Yunnan in China, Northern Vietnam) was recovered as a hybrid between Camellia fluviatilis (Guangdong, Guangxi and Hainan in China, Northeastern India, Northern Myanmar) and Camellia huana (Guangxi and Guizhou in China). The two putative parents show distribution overlap in Guangxi province of China, thus interspecies gene flow is likely to occur under natural conditions. Further work with increased taxon sampling will need to be conducted to uncover further introgression patterns among species within Camellia, an economically important genus of Theaceae.

\section{Whole genome duplication}

Two rounds of ancient WGD events, i.e. Ad- $\gamma$ and Ad- $\beta$, occurred in the tea tree (Camellia sinensis var. assamica) genome (Xia et al., 2017; Xia et al., 2020). However, analysis of genic collinearity reveals that a recent WGD event occurred after the divergence of tea and kiwifruit lineages, based on the genome of another variety of tea (Camellia sinensis var. sinensis) (Wei et al., 2018), Larson et al. (2020) named this WGD as Cm- $\alpha$. Recently, based on a chromosomescale genome of Camellia sinensis var. sinensis, the authors suggested one recent Camellia tetraploidization event occurred after the divergence of $C$. sinensis and A. chinensis from their common ancestor (Chen et al., 2020). But the time of the Camellia tetraploidization event (58.961.7 Ma) was very close to the divergence time between $C$. sinensis and A. chinensis at 61.2-65.3 Ma. Here, we identified a WGD event shared by all genera within Theaceae, and also other families such as Symplocaceae, Styracaceae, Pentaphylacaceae and Diapensiaceae (Fig. 4, Fig. S4), and also clarified that Cm- $\alpha$ proposed by Wei et al. (2018) and Larson et al. (2020) and the recent tetraploidization event found by Chen et al. (2020) were actually Ad- $\beta$ (i.e. Ericales clade). Ad- $\beta$ has been recently revised to the core Ericales clade according to genome 
collinearity and also MAPS pipeline (Leebens-Mack et al., 2019), and more specifically to the core Ericales+Primuloids+Polemonioids+Lecythidaceae clade, using deep Asterid phylotranscriptomic analyses (Zhang et al., 2020). Thus, our results support that the tea family experienced two WGD events in the evolutionary history, i.e. Ad- $\gamma$ and Ad- $\beta$, no other specific WGD event occurred within the family.

\section{Acknowledgement}

This work was supported by National Natural Science Foundation of China (No. 32070369, 31700182), the Large-scale Scientific Facilities of the Chinese Academy of Sciences (No. 2017LSFGBOWS-02), open Research Fund of Guangxi Key Laboratory of Special Non-wood Forest Cultivation \& Utilization (No. 19-B-01-03), the Youth Innovation Promotion Association CAS (No. 2021393) and CAS "Light of West China" Program. The authors are grateful to Prof. Liang Fang (Jiujiang Unversity), Prof. Zhong-Lang Wang, Drs. Jie Cai, Ting Zhang, Yun-Long Liu (Kunming Institute of Botany, Chinese Academy of Sciences) for their help with sample collection and data analysis, and to Mark Whitten, Sheng-Chen Shan (University of Florida) for their assistance for sampling of Stewartia malacodendron.

\section{References}

APG I. 1998. An ordinal classification for the families of flowering plants. Annals of the Missouri Botanical Garden 85: 531-553.

APG II. 2003. An update of the Angiosperm Phylogeny Group classification for the orders and families of flowering plants: APG II. Botanical Journal of the Linnean Society 141: 399-436.

APG III. 2009. An update of the Angiosperm Phylogeny Group classification for the orders and families of flowering plants: APG III. Botanical Journal of the Linnean Society 161: 105-121.

APG IV. 2016. An update of the Angiosperm Phylogeny Group classification for the orders and families of flowering plants: APG IV. Botanical Journal of the Linnean Society 181: 1-20.

Bentham G, Hooker J. 1862. Genera plantarum. Reeve, London.

Borowiec ML. 2016. AMAS: a fast tool for alignment manipulation and computing of summary statistics. Peerj 4: e1660.

Calvo J, Alvarez I, Aedo C, Pelser PB. 2013. A phylogenetic analysis and new delimitation of Senecio sect. Crociseris (Compositae: Senecioneae), with evidence of intergeneric hybridization. Taxon 62: 127140. 
Chang HD, Ren SX 1998. Theaceae (1). In: Wu CY ed. Flora Reipublicae Popularis Sinicae. Beijing: Science Press, 1-251.

Chang HT. 1976. Apterosperma-genus novum Theacearum. Acta Scientiarum Naturalium Universitatis Sunyatseni 15: 90-92.

Chen JD, Zheng C, Ma JQ, Jiang CK, Ercisli S, Yao MZ, Chen L. 2020. The chromosome-scale genome reveals the evolution and diversification after the recent tetraploidization event in tea plant. Horticulture Research 7: 63.

Folk RA, Mandel JR, Freudenstein JV. 2017. Ancestral Gene Flow and Parallel Organellar Genome Capture Result in Extreme Phylogenomic Discord in a Lineage of Angiosperms. Systematic Biology 66: 320337.

Folk RA, Soltis PS, Soltis DE, Guralnick R. 2018. New prospects in the detection and comparative analysis of hybridization in the tree of life. American Journal of Botany 105: 364-375.

Grabherr MG, Haas BJ, Yassour M, Levin JZ, Thompson DA, Amit I, et al. 2011. Full-length transcriptome assembly from RNA-Seq data without a reference genome. Nature Biotechnology 29: 644-652.

Grote PJ, Dilcher DL. 1992. Fruits and seeds of tribe Gordonieae (Theaceae) from the Eocene of North America. American Journal of Botany 79: 744-753.

Guo X, Thomas D, Saunders R. 2018. Gene tree discordance and coalescent methods support ancient intergeneric hybridisation between Dasymaschalon and Friesodielsia (Annonaceae). Molecular Phylogenetics and Evolution 127: 14-29.

Haas BJ, Papanicolaou A, Yassour M, Grabherr M, Blood PD, Bowden J, et al. 2013. De novo transcript sequence reconstruction from RNA-seq using the Trinity platform for reference generation and analysis. Nature Protocols 8: 1494-1512.

Huang CH, Sun R, Hu Y, Zeng L, Zhang N, Cai L, Zhang Q, Koch MA, Al-Shehbaz I, Edger PP, Pires JC, Tan DY, Zhong Y, Ma H. 2015. Resolution of Brassicaceae Phylogeny Using Nuclear Genes Uncovers Nested Radiations and Supports Convergent Morphological Evolution. Molecular Biology and Evolution 33: 394-412.

Huang CH, Zhang CF, Liu M, Hu Y, Gao TG, Qi J, Ma H. 2016. Multiple Polyploidization Events across Asteraceae with Two Nested Events in the Early History Revealed by Nuclear Phylogenomics. Molecular Biology and Evolution 33: 2820-2835.

Huang SX, Ding J, Deng DJ, Tang W, Sun HH, Liu DY, Zhang L, Niu XL, Zhang X, Meng M. 2013. Draft genome of the kiwifruit Actinidia chinensis. Nature Communications 4: 2640.

IUCN 2020. The IUCN Red List of Threatened Species, Version 2020-1.

Jiao YN, Wickett NJ, Ayyampalayam S, Chanderbali AS, Landherr L, Ralph PE, et al. 2011. Ancestral polyploidy in seed plants and angiosperms. Nature 473: 97-100.

Jin JJ, Yu WB, Yang JB, Song Y, dePamphilis CW, Yi TS, Li DZ. 2020. GetOrganelle: a fast and versatile toolkit for accurate de novo assembly of organelle genomes. Genome biology 21: 241.

Katoh K, Standley DM. 2013. MAFFT multiple sequence alignment software version 7: improvements in performance and usability. Molecular Biology and Evolution 30: 772-780.

Keng H. 1980. On the unification of Laplacea and Gordonia (Theaceae). Gardens' Bulletin (Singapore) 33: 303-311.

Kobuski CE. 1949. Studies in the Theaceae XVIII. The West Indian species of Laplacea. Journal of the Arnold Arboretum 30: 166-186.

Kobuski CE. 1950. Studies in the Theaceae. XX. Notes on the South and Central American species of Laplacea. Journal of the Arnold Arboretum 31: 405-429.

Kvaček Z. 2004. Revisions to the Early Oligocene flora of Flörsheim (Mainz Basin, Germany) based on epidermal anatomy. Senckenbergiana lethaea 84: 1-73.

Kvacek Z, Walther H. 1998. The Oligocene Volcanic Flora of Kundratice near Litomerice, Ceske Stredohori Volcanic Complex (Czech Republic)-A review. Sbornik Narodniho Muzea v Praze, Rada B - Prirodni Vedy (Acta Musei Nationalis Pragae, Series B, Historia Naturalis) 54: 1-42.

Lanfear R, Frandsen PB, Wright AM, Senfeld T, Calcott B. 2016. PartitionFinder 2: New Methods for Selecting Partitioned Models of Evolution for Molecular and Morphological Phylogenetic Analyses. Molecular Biology and Evolution 34: 772-773. 
Larson DA, Walker JF, Vargas OM, Smith SA. 2020. A consensus phylogenomic approach highlights paleopolyploid and rapid radiation in the history of Ericales. American Journal of Botany 107: 1-17.

Lee SL, Yang TYA. 2019. Camellia chinmeii, a new species of Camellia sect. Paracamellia in Taiwan. Taiwania 64: 321-325.

Leebens-Mack JH, Barker MS, Carpenter EJ, Deyholos MK, Gitzendanner MA, Graham SW, et al., One Thousand Plant T. 2019. One thousand plant transcriptomes and the phylogenomics of green plants. Nature 574: 679-685.

Li MM, Li JH, Del Tredici P, Corajod J, Fu CX. 2013. Phylogenetics and biogeography of Theaceae based on sequences of plastid genes. Journal of Systematics and Evolution 51: 396-404.

Li MM, Meegahakumbura Kasun M, Yan LJ, Liu J, Gao LM. 2015. Genetic Involvement of Camellia taliensis in the Domestication of $C$. sinensis var. assamica (Assimica Tea) Revealed by Nuclear Microsatellite Markers. Plant Diversity and Resourcues 37: 29-37.

Li W, Godzik A. 2006. Cd-hit: a fast program for clustering and comparing large sets of protein or nucleotide sequences. Bioinformatics 22: 1658-1659.

Lin HY, Hao YJ, Li JH, Fu CX, Soltis PS, Soltis DE, Zhao YP. 2019. Phylogenomic conflict resulting from ancient introgression following species diversification in Stewartia s.l. (Theaceae). Molecular Phylogenetics and Evolution 135: 1-11.

Liu ZW, Fang W, Liu ED, Zhao M, He YF, Yang SX. 2019. Camellia mingii, a new species of yellow camellias from Southeast Yunnan, China. Phytotaxa 393: 47.

Matasci N, Hung LH, Yan ZX, Carpenter EJ, Wickett NJ, Mirarab S, et al. 2014. Data access for the 1,000 Plants (1KP) project. Gigascience 3: 17.

Meegahakumbura MK, Wambulwa MC, Thapa KK, Li MM, Moller M, Xu JC, et al. 2016. Indications for Three Independent Domestication Events for the Tea Plant (Camellia sinensis (L.) O. Kuntze) and New Insights into the Origin of Tea Germplasm in China and India Revealed by Nuclear Microsatellites. PLOS ONE 11: e0155369.

Melchior H 1925. Theaceae. In: Engle A, Prantl E eds. Die naturlichen Pflanzenfamilien, 2nd ed. Leipzig, Germany: Wilhelm Engelmann, 109-154.

Milne RI. 2006. Northern Hemisphere plant disjunctions: a window on tertiary land bridges and climate change? Annals of Botany 98: 465-472.

Min TL, Bartholomew B 2007. Theaceae. In: Wu CY, Raven PH eds. Flora of China. Beijing, China and Saint Louis, Missouri, USA: Science Press and Missouri Botanical Garden Press, 366-478.

Mirarab S, Reaz R, Bayzid MS, Zimmermann T, Swenson MS, Warnow T. 2014. ASTRAL: genomescale coalescent-based species tree estimation. Bioinformatics 30: i541-i548.

Mirbel CFB. 1813. Notes pour servir a l'histoire naturelle de la famille des Orangers de M. A.-L. de Jussieu. Nouveau Bulletin des Sciences par la Société Philomatique 75: 382.

Morales-Briones DF, Liston A, Tank DC. 2018. Phylogenomic analyses reveal a deep history of hybridization and polyploidy in the Neotropical genus Lachemilla (Rosaceae). New Phytologist 218: $1668-1684$.

Nishimoto S, Shimizu K, Hashimoto F, Sakata Y. 2003. Interspecific hybrids of Camellia chrysantha x $C$. japonica by ovule culture. Journal of the Japanese Society for Horticultural Science 72: 236-242.

Orel G. 2006. A new species of Camellia section Piquetia (Theaceae) from Vietnam. Novon 16: 244-247.

Orel G, Wilson PG. 2010a. Camellia luteocerata sp. nov. and a new section of Camellia (Dalatia) from Vietnam. Nordic Journal of Botany 28: 280-284.

Orel G, Wilson PG. 2010b. A New Species of Camellia Sect. Stereocarpus (Theaceae) from Vietnam. Novon 20: 198-202.

Orel G, Wilson PG. 2012. Camellia cherryana (Theaceae), a new species from China. Annales Botanici Fennici 49: 248-254.

Orel G, Wilson PG, Curry AS. 2014. Four New Species and Two New Sections of Camellia (Theaceae) from Vietnam. Novon 23: 307-318.

Orel G, Wilson PG, Curry AS, Luu HT. 2013. Two new species of Polyspora (Theaceae) from Vietnam and new combinations for some Asian species. Willdenowia-Annals of the Botanic Garden and Botanical Museum Berlin-Dahlem 43: 301-308. 
Prince LM 1993. Theaceae. In: Committee FoNAE ed. Flora of North America. New York and Oxford: Oxford University Press, 322-323.

Prince LM, Parks CR. 2001. Phylogenetic relationships of Theaceae inferred from chloroplast DNA sequence data. American Journal of Botany 88: 2309-2320.

Qiao X, Li Q, Yin H, Qi K, Li L, Wang R, Zhang S, Paterson AH. 2019. Gene duplication and evolution in recurring polyploidization-diploidization cycles in plants. Genome biology 20: 38.

Ran JH, Shen TT, Wu H, Gong X, Wang XQ. 2018. Phylogeny and evolutionary history of Pinaceae updated by transcriptomic analysis. Molecular Phylogenetics and Evolution 129: 106-116.

Ronquist F, Teslenko M, van der Mark P, Ayres DL, Darling A, Hohna S, Larget B, Liu L, Suchard MA, Huelsenbeck JP. 2012. MrBayes 3.2: efficient Bayesian phylogenetic inference and model choice across a large model space. Systematic Biology 61: 539-542.

Rose JP, Kleist TJ, Lofstrand SD, Drew BT, Schonenberger J, Sytsma KJ. 2018. Phylogeny, historical biogeography, and diversification of angiosperm order Ericales suggest ancient Neotropical and East Asian connections. Molecular Phylogenetics and Evolution 122: 59-79.

Sayyari E, Mirarab S. 2016. Fast Coalescent-Based Computation of Local Branch Support from Quartet Frequencies. Molecular Biology and Evolution 33: 1654-1668.

Shi T, Huang HW, Barker MS. 2010. Ancient genome duplications during the evolution of kiwifruit (Actinidia) and related Ericales. Annals of Botany 106: 497-504.

Smith SA, Moore MJ, Brown JW, Yang Y. 2015. Analysis of phylogenomic datasets reveals conflict, concordance, and gene duplications with examples from animals and plants. BMC Evolutionary Biology 15: 150.

Soltis DE, Gitzendanner MA, Stull G, Chester M, Chanderbali A, Chamala S, Jordon-Thaden I, Soltis PS, Schnable PS, Barbazuk WB. 2013. The potential of genomics in plant systematics. Taxon 62: 886-898.

Stamatakis A. 2014. RAxML version 8: a tool for phylogenetic analysis and post-analysis of large phylogenies. Bioinformatics 30: 1312-1313.

Stevens PF. 2001 onwards. Angiosperm Phylogeny Website. http://www.mobot.org/MOBOT/research/APweb/.

Stubbs RL, Folk RA, Xiang C-L, Chen S, Soltis DE, Cellinese N. 2020. A Phylogenomic Perspective on Evolution and Discordance in the Alpine-Arctic Plant Clade Micranthes (Saxifragaceae). Frontiers in Plant Science 10: 1773.

Takhtajan AL. 1997. Diversity and the Classification of Flowering Plants. New York, USA: Columbia University Press.

Tanaka T, Mizutani T, Shibata M, Tanikawa N, Parks CR. 2005. Cytogenetic studies on the origin of Camellia $\mathrm{x}$ vernalis. V. estimation of the seed parent of $C$. $\mathrm{x}$ vernalis that evolved about 400 years ago by cpDNA analysis. Journal of the Japanese Society for Horticultural Science 74: 464-468.

Tang CQ 2015. Evergreen Broad-Leaved Forests. In: Tang CQ ed. The Subtropical Vegetation of Southwestern China: Plant Distribution, Diversity and Ecology Utrecht, The Netherlands: Springer Netherlands, 49-112.

Than C, Ruths D, Nakhleh L. 2008. PhyloNet: a software package for analyzing and reconstructing reticulate evolutionary relationships. BMC Bioinformatics 9: 322.

Tiffney BH. 1985. Perspectives on the origin of the floristic similarity between Eastern Asia and Eastern North-America. Journal of the Arnold Arboretum 66: 73-94.

Tiffney BH, Manchester SR. 2001. The use of geological and paleontological evidence in evaluating plant phylogeographic hypotheses in the Northern Hemisphere tertiary. International Journal of Plant Sciences 162: S3-S17.

Tsou CH. 1998. Early floral development of Camellioideae (Theaceae). American Journal of Botany 85 : 1531-1547.

Vekemans D, Proost S, Vanneste K, Coenen H, Viaene T, Ruelens P, Maere S, Van de Peer Y, Geuten K. 2012. Gamma Paleohexaploidy in the Stem Lineage of Core Eudicots: Significance for MADS-Box Gene and Species Diversification. Molecular Biology and Evolution 29: 3793-3806. 
Wang YH, He H, Min TL, Zhou LH, Fritsch PW. 2006. The phylogenetic position of Apterosperma (Theaceae) based on morphological and karyotype characters. Plant Systematics and Evolution 260: $39-52$.

Wei CL, Yang H, Wang S, Zhao J, Liu C, Gao L, et al. 2018. Draft genome sequence of Camellia sinensis var. sinensis provides insights into the evolution of the tea genome and tea quality. Proceedings of the National Academy of Sciences of the United States of America 115: E4151-E4158.

Wickett NJ, Mirarab S, Nguyen N, Warnow T, Carpenter E, Matasci N, et al. 2014. Phylotranscriptomic analysis of the origin and early diversification of land plants. Proceedings of the National Academy of Sciences 111: E4859-4868.

Wilde V, Frankenhauser H. 1998. The Middle Eocene plant taphocoenosis from Eckfeld (Eifel, Germany). Review of Palaeobotany and Palynology 101: 7-28.

Xia EH, Tong W, Hou Y, An YL, Chen LB, Wu Q, et al. 2020. The reference genome of tea plant and resequencing of 81 diverse accessions provide insights into genome evolution and adaptation of tea plants. Molecular Plant 13: 1013-1026.

Xia EH, Zhang HB, Sheng J, Li K, Zhang QJ, Kim C, et al. 2017. The Tea Tree Genome Provides Insights into Tea Flavor and Independent Evolution of Caffeine Biosynthesis. Molecular Plant 10: 866-877.

Xiang YZ, Huang CH, Hu Y, Wen J, Li SS, Yi TS, Chen HY, Xiang J, Ma H. 2017. Evolution of Rosaceae fruit types based on nuclear phylogeny in the context of geological times and genome duplication. Molecular Biology and Evolution 34: 262-281.

Xu XD, Zheng W, Harris A, Wang W, Shao WZ, Wen J. 2018. Assessing the maternal origin in the polyploid complex of Camellia reticulata based on the chloroplast rpl16 intron sequences: implications for camellia cross breeding. Molecular Breeding 38: 123.

Yang JB, Yang SX, Li DZ, Lei LG, Ikeda T, Yoshno H. 2006. Phylogenetic relationships of Theaceae inferred from mitochondrial matR Gene sequence data. Acta Botanica Yunnanica 28: 29-36.

Yang SX, Yang JB, Lei LG, Li DZ, Yoshino H, Ikeda T. 2004. Reassessing the relationships between Gordonia and Polyspora (Theaceae) based on the combined analyses of molecular data from the nuclear, plastid and mitochondrial genomes. Plant Systematics and Evolution 248: 45-55.

Yang Y, Moore MJ, Brockington SF, Soltis DE, Wong GKS, Carpenter EJ, Zhang Y, Chen L, Yan ZX, Xie YL. 2015. Dissecting molecular evolution in the highly diverse plant clade Caryophyllales using transcriptome sequencing. Molecular Biology and Evolution 32: 2001-2014.

Yang Y, Smith SA. 2013. Optimizing de novo assembly of short-read RNA-seq data for phylogenomics. Bmc Genomics 14: 328.

Yang Y, Smith SA. 2014. Orthology inference in nonmodel organisms using transcriptomes and low-coverage genomes: improving accuracy and matrix occupancy for phylogenomics. Molecular Biology and Evolution 31: 3081-3092.

Ye CX. 1990. A discussion on relationship among the genera in Theoideae (Theaceae). Acta Scientiarum Naturalium Universitatis Sunyatseni 29: 74-81.

Yu XQ, Drew BT, Yang JB, Gao LM, Li DZ. 2017a. Comparative chloroplast genomes of eleven Schima (Theaceae) species: Insights into DNA barcoding and phylogeny. PLoS ONE 12: e0178026.

Yu XQ, Gao LM, Soltis DE, Soltis PS, Yang JB, Fang L, Yang SX, Li DZ. 2017b. Insights into the historical assembly of East Asian subtropical evergreen broadleaved forests revealed by the temporal history of the tea family. New Phytologist 215: 1235-1248.

Yu XQ, Yang D, Guo C, Gao LM. 2018. Plant phylogenomics based on genome-partitioning strategies: Progress and prospects. Plant diversity 40: 158-164.

Yu Y, Nakhleh L. 2015. A maximum pseudo-likelihood approach for phylogenetic networks. Bmc Genomics 16(Suppl 10): S10.

Zeng LP, Zhang N, Zhang Q, Endress PKH, Jie, Ma H. 2017. Resolution of deep eudicot phylogeny and their temporal diversification using nuclear genes from transcriptomic and genomic datasets. New Phytologist 214: 1338-1354.

Zeng LP, Zhang Q, Sun RR, Kong HZ, Zhang N, Ma H. 2014. Resolution of deep Angiosperm phylogeny using conserved nuclear genes and estimates of early divergence times. Nature Communications $\mathbf{5}$ : 4956. 
Zhang CF, Zhang TK, Luebert F, Xiang YZ, Huang CH, Hu Y, Rees M, Frohlich MW, Qi J, Weigend M, Ma H. 2020. Asterid phylogenomics/phylotranscriptomics uncover morphological evolutionary histories and support phylogenetic placement for numerous whole genome duplications. Molecular Biology and Evolution 37: 3188-3210.

Zhang W, Kan SL, Zhao H, Li ZY, Wang XQ. 2014. Molecular Phylogeny of Tribe Theeae (Theaceae s.s.) and Its Implications for Generic Delimitation. PLOS ONE 9: e98133.

Zhao L, Li X, Zhang N, Zhang SD, Yi TS, Ma H, Guo ZH, Li DZ. 2016. Phylogenomic analyses of largescale nuclear genes provide new insights into the evolutionary relationships within the rosids. Molecular Phylogenetics and Evolution 105: 166-176.

Zwaenepoel A, Van de Peer Y. 2019. wgd-simple command line tools for the analysis of ancient wholegenome duplications. Bioinformatics 35: 2153-2155. 


\section{Figure legend}

Figure 1 Phylogeny of Theaceae inferred from partitioned maximum likelihood (ML) analysis of the concatenated 610 low-copy nuclear genes, numbers associated with nodes indicate ML bootstrap support (BS)/Bayesian inference (BI) posterior probability (PP) values. Asterisks represent nodes with $100 \%$ support from both analyses.

Figure 2 Patterns of gene-tree concordance and conflict of Theaceae based on the PhyParts analysis. The tree topology used was that inferred by ASTRAL. The pie charts at each node show the proportion of genes in concordance (blue), conflict (green $=$ a single dominant alternative; red = all other conflicting trees), and without enough information (gray). The numbers above and below each branch are the numbers of concordant and conflicting genes at each bipartition, respectively.

Figure 3 The optimal phylogenetic network of Theaceae inferred using PhyloNet, with the number of reticulations as one, two, three and four. The scenario with four reticulations had the best $\log$ pseudo-likelihood.

Figure $4 K s$ distribution for paralogs with mixture models of inferred WGDs (blue dashed line) for Apterosperma oblata, Camellia fluviatilis, Polyspora hainanensis, Schima superba, Stewartia pteropetiolata and Pyrenaria jonquieriana subsp. multisepala. 
Table 1 List of taxa sampled in this study, with voucher and Illumina reads, species names with underscore represent those species selected for PhyloNet analysis

\begin{tabular}{|c|c|c|c|c|c|}
\hline Taxon & $\begin{array}{l}\text { Voucher } \\
\text { specimen }\end{array}$ & Sources & $\begin{array}{l}\text { No. reads } \\
\text { (trimmed) }\end{array}$ & SRA number & $\begin{array}{l}\text { Plastid } \\
\text { genome }\end{array}$ \\
\hline \multicolumn{6}{|l|}{ Stewartieae } \\
\hline Stewartia calcicola & YXQ090 & Yunnan, China & $76,314,960$ & - & KY406783 \\
\hline Stewartia cordifolia & YXQ144 & Guangxi, China & $101,158,084$ & - & KY406775 \\
\hline Stewartia crassifolia & YXQ171 & Hunan, China & $88,878,696$ & - & KY406766 \\
\hline Stewartia malacodendron & FLAS 260361 & Alabama, USA & $1,798,360,616$ & - & KY406773 \\
\hline Stewartia ovata & $18847 * \mathrm{~A}$ & The Arnold Arboretum & $100,544,128$ & - & KY406782 \\
\hline Stewartia pseudocamellia & MO-6587810 & $\begin{array}{l}\text { Missouri Botanical } \\
\text { Garden }\end{array}$ & $83,044,916$ & - & KY406786 \\
\hline Stewartia pteropetiolata & YXQ038 & Yunnan, China & $124,092,532$ & - & KY406770 \\
\hline Stewartia rostrata & YXQ15072003 & Jiangxi, China & $91,741,812$ & - & KY406789 \\
\hline Stewartia rubiginosa & YXQ189 & Hunan, China & $91,059,484$ & - & KY406777 \\
\hline $\begin{array}{l}\text { Stewartia sinensis } \\
\text { Gordonieae }\end{array}$ & YXQ15072001 & Jiangxi, China & $91,325,600$ & - & KY406748 \\
\hline $\begin{array}{l}\text { Gordonieae } \\
\text { Franklinia alatamaha } \\
\end{array}$ & MO-6587811 & $\begin{array}{l}\text { Missouri Botanical } \\
\text { Garden }\end{array}$ & $101,387,448$ & - & KY406774 \\
\hline Gordonia brandegeei & N. Zamora 7196 & Guanacaste, Costa Rica & $104,462,244$ & - & KY406761 \\
\hline$\overline{\text { Gordonia lasianthus }}$ & JCRA 110687 & $\begin{array}{l}\text { JC Raulston } \\
\text { Arboretum, Raleigh, } \\
\text { North Carolina }\end{array}$ & $82,908,172$ & - & KY406790 \\
\hline Schima argentea & YXQ226 & Yunnan, China & $86,065,752$ & - & - \\
\hline Schima brevipedicellata & YXQ072 & Yunnan, China & $89,409,700$ & - & KY406784 \\
\hline Schima noronhae & YXQ034 & Yunnan, China & $88,824,452$ & - & KY406787 \\
\hline Schima sericans & YXQ053 & Yunnan, China & $97,108,488$ & - & KY406779 \\
\hline Schima superba & YXQ142 & Guangxi, China & $91,704,880$ & - & KY406788 \\
\hline Schima wallichii & YXQ001 & Yunnan, China & $89,876,764$ & - & KY406795 \\
\hline \multicolumn{6}{|l|}{ Theeae } \\
\hline Apterosperma oblata & YangSX 5978 & Guangdong, China & $86,466,584$ & - & - \\
\hline Camellia amplexifolia & YangSX 5010 & Hainan, China & $98,672,716$ & - & - \\
\hline Camellia assimiloides & YangSX 5540 & Guangdong, China & $98,123,144$ & - & - \\
\hline Camellia cordifolia & YangSX 5551 & Guangdong, China & $96,616,940$ & - & - \\
\hline Camellia cuspidata & YangSX 5118 & Hubei, China & $78,369,336$ & - & - \\
\hline Camellia flavida & YangSX 5865 & Guangxi, China & $92,242,904$ & - & - \\
\hline Camellia fluviatilis & YangSX 4033 & Guangxi, China & $68,780,488$ & - & - \\
\hline$\overline{\text { Camellia grijsii }}$ & YangSX 6064 & Guizhou, China & $120,959,328$ & - & - \\
\hline Camellia gymnogyna & YangSX 5953 & Guangxi, China & $94,768,804$ & - & - \\
\hline Camellia huana & YangSX 5653 & Guizhou, China & $111,316,580$ & - & - \\
\hline Camellia ilicifolia & YangSX 5287 & Guizhou, China & $95,559,812$ & - & - \\
\hline Camellia longipedicellata & YangSX 5926 & Guangxi, China & $90,347,792$ & - & - \\
\hline Camellia longissima & YangSX 5079 & Guangxi, China & $99,050,172$ & - & - \\
\hline Camellia luteoflora & YangSX 6063 & Guizhou, China & $123,788,308$ & - & - \\
\hline Camellia pilosperma & YangSX 4714 & Guangxi, China & $92,105,712$ & - & - \\
\hline Camellia pitardii var. compressa & YangSX 4576 & Hunan, China & $76,531,640$ & - & - \\
\hline Camellia semiserrata & YangSX 5555 & Guangdong, China & $86,751,660$ & - & - \\
\hline Camellia sinensis var. pubilimba & YangSX 5927 & Guangxi, China & $85,785,132$ & - & - \\
\hline Camellia szechuanensis & YangSX 5064 & Sichuan, China & $88,726,296$ & - & - \\
\hline$\overline{\text { Camellia tsingpienensis }}$ & YangSX 5798 & Guangxi, China & $90,514,420$ & - & - \\
\hline$\overline{\text { Camellia tuberculata }}$ & YangSX 5202 & Chongqing, China & $90,076,900$ & - & - \\
\hline Laplacea fruticosa & NZ10477 & Puntarenas, Costa Rica & $1,532,313,620$ & - & - \\
\hline Polyspora axillaris & YXQ099 & Hainan, China & $92,917,500$ & - & KY406760 \\
\hline Polyspora chrysandra & YXQ221 & Yunnan, China & $84,011,964$ & - & - \\
\hline
\end{tabular}




\begin{tabular}{|c|c|c|c|c|c|}
\hline Polyspora dalgleishiana & BROWP 501 & $\begin{array}{l}\text { Royal Botanic Garden } \\
\text { Edinburgh }\end{array}$ & $83,838,300$ & - & KY406769 \\
\hline Polyspora hainanensis & YXQ097 & Hainan, China & $97,170,208$ & - & KY406776 \\
\hline$\overline{\text { Polyspora longicarpa }}$ & YangSX 4779 & Yunnan, China & $89,251,900$ & - & KY406768 \\
\hline Polyspora speciosa & YXQ145 & Guangxi, China & $96,508,612$ & - & KY406754 \\
\hline Pyrenaria hirta var. cordatula & YXQ169 & Guangxi, China & $92,426,012$ & - & KY406785 \\
\hline Pyrenaria hirta var. hirta & YangSX 4067 & Guangxi, China & $100,342,800$ & - & - \\
\hline $\begin{array}{l}\text { Pyrenaria jonquieriana } \\
\text { subsp. multisepala }\end{array}$ & YXQ106 & Hainan, China & $87,583,392$ & - & KY406772 \\
\hline Pyrenaria khasiana & YangSX 5046 & Myanmar, Kachin & $88,528,812$ & - & KY406756 \\
\hline Pyrenaria menglaensis & YXQ211 & Yunnan, China & $87,085,244$ & - & KY406747 \\
\hline $\begin{array}{l}\text { Pyrenaria microcarpa var. micro } \\
\text { carpa }\end{array}$ & YXQ101 & Hainan, China & $81,080,788$ & - & KY406764 \\
\hline Pyrenaria oblongicarpa & YXQ216 & Yunnan, China & $98,658,408$ & - & KY406781 \\
\hline Pyrenaria pingpienensis & YXQ210 & Yunnan, China & $96,601,500$ & - & - \\
\hline Pyrenaria shinkoensis & YangSX 5038 & Taiwan, China & $101,120,720$ & - & - \\
\hline $\begin{array}{l}\text { Pyrenaria spectabilis var. greeni } \\
\text { ae }\end{array}$ & YXQ172 & Hunan, China & $93,451,072$ & - & KY406753 \\
\hline $\begin{array}{l}\text { Pyrenaria spectabilis var. specta } \\
\text { bilis }\end{array}$ & YXQ155 & Guangxi, China & $88,012,020$ & - & KY406765 \\
\hline \multicolumn{6}{|l|}{$\overline{\text { Outgroup }}$} \\
\hline Eurya acuminatissima & - & - & $25,009,135$ & SRX2786652 & - \\
\hline Galax urceolata & - & - & $9,647,946$ & ERX2099546 & - \\
\hline Sinojackia xylocarpa & - & - & $10,611,525$ & ERX2099565 & - \\
\hline Symplocos tinctoria & - & - & $10,196,542$ & ERX2099566 & - \\
\hline Symplocos paniculata & - & - & $28,374,406$ & SRX1601992 & - \\
\hline Ternstroemia gymnanthera & - & - & $14,421,549$ & ERX2099558 & - \\
\hline
\end{tabular}
-represents the chloroplast genes were extracted from the transcriptome data or no data were available.

Table 2 Total $\log$ probability of each phylogenetic network

\begin{tabular}{ll}
\hline Reticulations & Total log probability \\
\hline 1 & -380.3215 \\
2 & -380.3215 \\
3 & -380.1597 \\
4 & -379.6899 \\
\hline
\end{tabular}




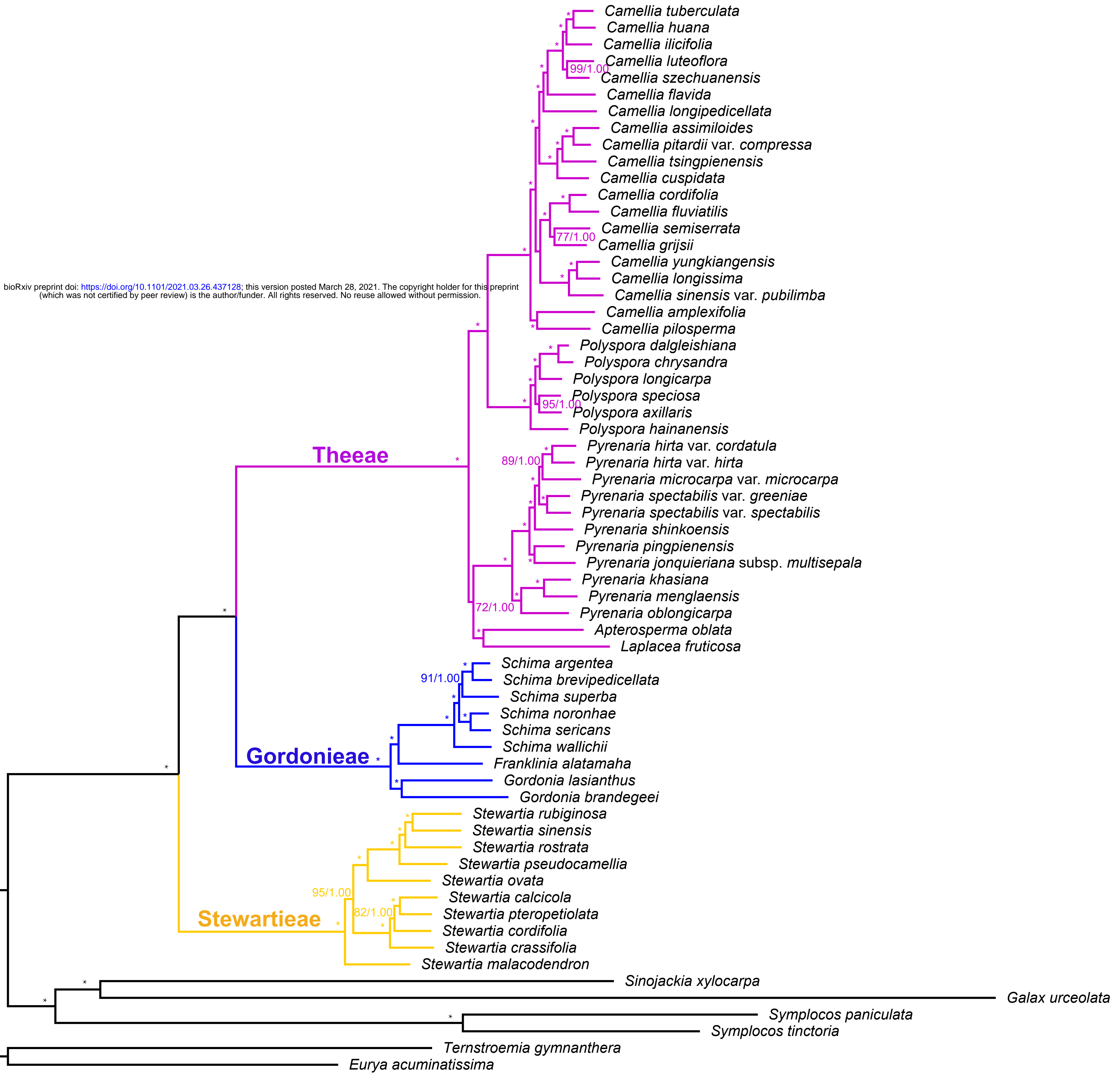



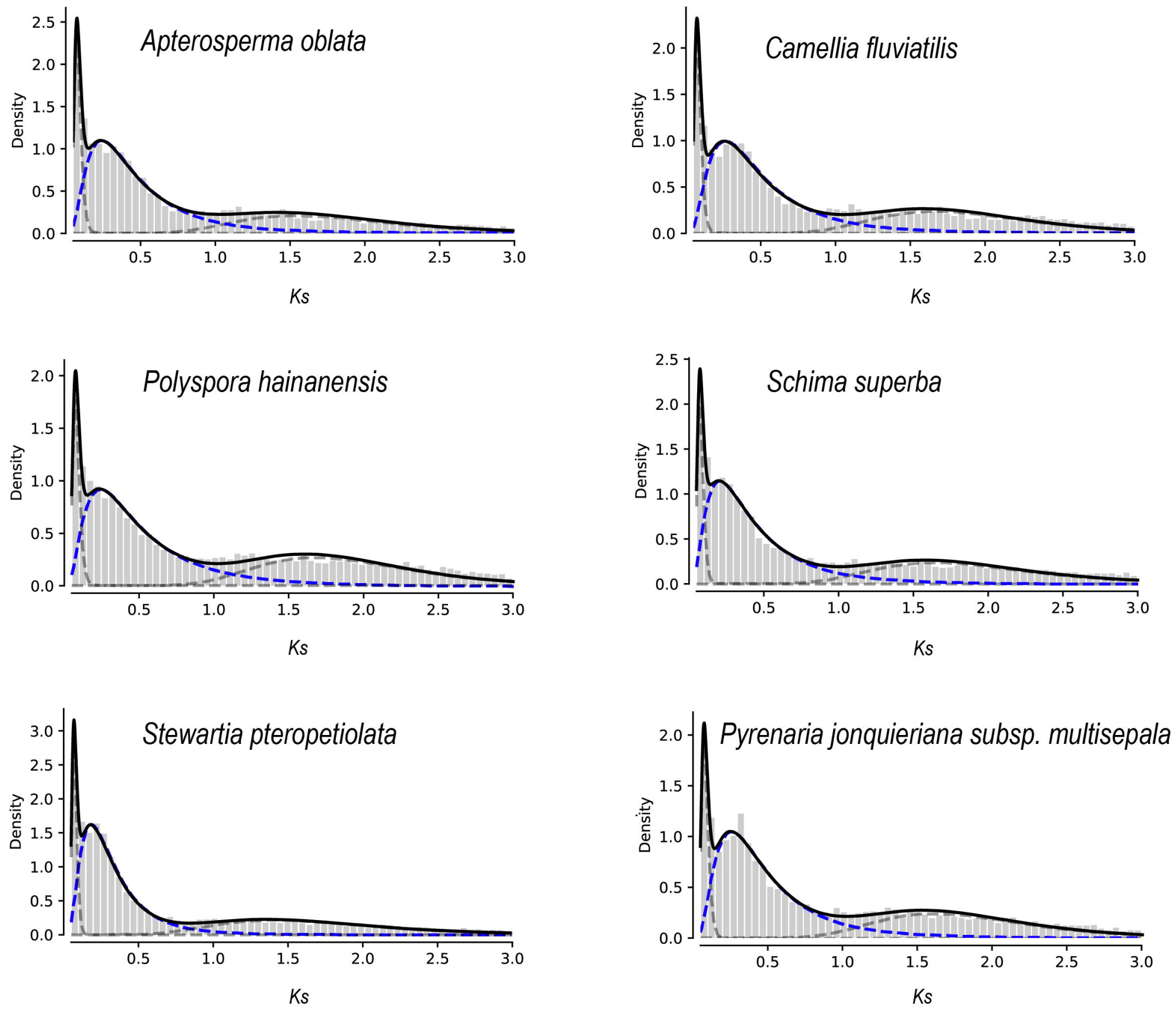